\title{
Directional emissivity correction by photogrammetric 3D object reconstruction
}

\author{
by P. Sawicki ${ }^{1}$, R. Stein ${ }^{2}$ and B. Więcek ${ }^{2}$
}

${ }^{1}$ Olsztyn University, Department of Photogrammetry and Remote Sensing, Oczapowskiego 1, 10-957

Olsztyn,Poland, e-mail: sawicki@kfit.uni.olsztyn.pl

${ }^{2}$ Technical University of Łódź, Institute of Electronics, Computer Thermography Group 18/22

Stefanowskiego St, 90-924 Łódż, Poland, e-mail: wiecek@ck-sg.p.lodz.pl

\begin{abstract}
In this paper, the detailed algorithm for directional emissivity correction by 3D object reconstruction is presented. The method is based on photogrammetric Direct Linear Transformation (DLT) with numerical improvements to achieve better convergence. The results of DLT solution are used to correct the directional emissivity and introduce the distance dependent transmission of ambient. Presented algorithm was practically tested in multichannel video-thermal system with one thermal and four optical channels. The algorithm is useful in various applications, e.g.: in industrial photogrammetric monitoring, optical metrology, robotics.
\end{abstract}

\section{Introduction}

The 3D object reconstruction is widely used in photogrammetric applications. Up to now $3 \mathrm{D}$ reconstruction was applied effectively with digital video images. It seems that combining video and thermal systems together with $3 \mathrm{D}$ image reconstruction implemented, provides new possibilities for thermography. Localising an object in 3D space directly allows to evaluate a distance and orientation of thermal specimen, and correct object's emissivity and ambient transmission for infrared radiation.

There are well known methods of 3D object reconstruction [1,5]. A method called Shape from Shading and Direct Linear Transformation are the most exploited ones $[1,2,5]$. The numerical complexity and a huge amount of information to be processed were the main obstacle for a practical implementation of 3D image reconstruction. New computer technology allows to realise systems for such processing today $[3,4]$.

Direct Linear Transformation method requires at least two CCD cameras. DLT describes position of $3 \mathrm{D}$ point on both photos, what allows to compute 3D position by reciprocal transformation using images coordinates. This method gives all information about 3D position of the point, including distance between the camera and the point and the angle between normal to the surface and the axes of the camera. It allows all kind of corrections of thermal images, because the dependence between emissivity and the inclination and distance are well known. Unlike the first method, the position of the point is very accurate and precisely computed, so this algorithm is very helpful in all high precision applications.

In this paper we present directional emissivity correction of thermal images for application in microelectronics. The temperature for complex-shape radiator is recalculated to get the more precise temperature distribution.

\section{Principle of the method}

A camera setup for 3D object reconstruction is shown Fig.1. We need to define 3D coordinates of 6 points from each visual image. These six points on each video images (called later control points) are used for video CCD camera positioning, needed for future coordinates' calculating for unknown points. After positioning video CCD cameras, one should marked any extra 6 pass points on both video and thermal images. These extra 6 points may be the same or different as control points used for CCD cameras positioning. Program automatically calculates coordinates of this extra pass points and compute position 
of thermal camera without any given coordinates of marked control points on thermal image. This procedure is helpful when it is difficult to locate on the thermal image any of 6 control points. On the other hand, one can recognize the same points on each image, including the thermal one, without providing their real coordinates. In the final stage of the algorithm, groups of 3 points marked by the user are used to determine the chosen planes for which, angle and distance are calculated, and used later for emissivity and temperature correction.

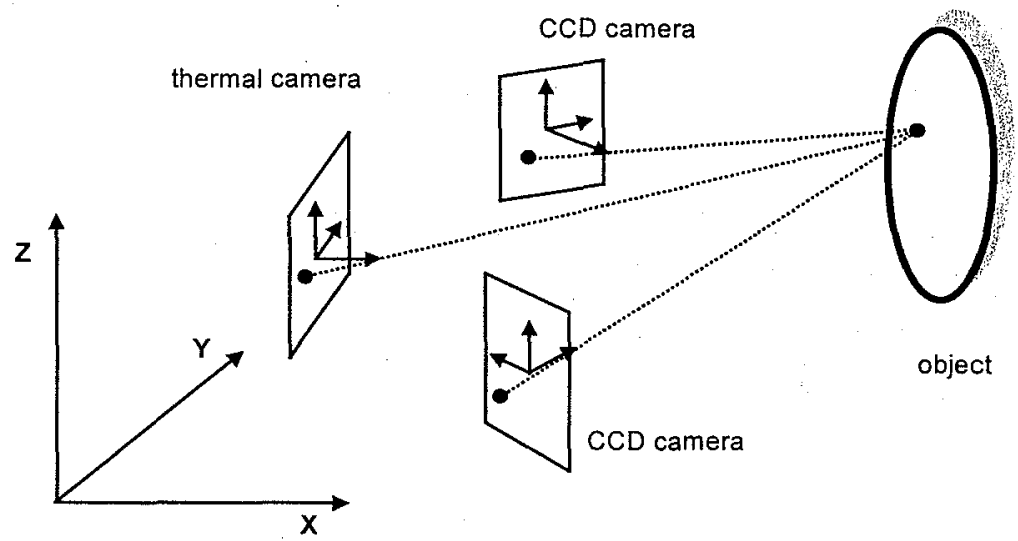

Fig. 1. Camera setup for 3-D object reconstruction

Each camera gives a different view of an observed object. Each object point visible on both photos has its own (different for each one) 2D image position. Differences between this location on images obtained from different cameras position are the base for computing the $3 \mathrm{D}$ object points positioning. The image is a result of transformation of 3D object into a flat surface of a CCD sensor in the camera. This transformation is affine what makes possible to present the dependence between the coordinates of point in $3 \mathrm{D}$ space and in $2 \mathrm{D}$ image in the form:

$$
\left[\begin{array}{c}
x^{*}-x_{p} \\
y^{*}-y_{p} \\
-c
\end{array}\right]=S\left[\begin{array}{lll}
m_{11} & m_{12} & m_{13} \\
m_{21} & m_{22} & m_{23} \\
m_{31} & m_{32} & m_{33}
\end{array}\right]\left[\begin{array}{c}
X-X_{0} \\
Y-Y_{0} \\
Z-Z_{0}
\end{array}\right]
$$

where:

$\left(x^{*}, y^{*}\right)$ - measured image point coordinates,

$\left(x_{p}, y_{p}\right)$ - coordinates of principle point offset,

$c$ - focal lenght, $S$ - scale factor,

$\bar{m}$ - matrix of rotation (parameters of camera exterior orientation),

$\left(\begin{array}{lll}X & Y & Z\end{array}\right)^{T}-3 \mathrm{D}$ coordinates of point in reference to chosen $\mathrm{XYZ}$ set

$\left(\begin{array}{lll}X_{0} & Y_{0} & Z_{0}\end{array}\right)^{T}$ - vector of translation (camera exterior orientation)

The last equation describes completely the way of the ray in camera's lens. Typically, two or more video cameras take photos of the same object, so we can write set of such equation for each point and camera, and solve it to get $3 \mathrm{D}$ coordinates. But first, this equation must be solved for all unknown values describing the exterior orientation parameters and other interior orientation parameters of the camera. These parameters will 
be described in details below. We apply $D L T$ theory, where each point's transformation is described by eqn. (2):

$$
\begin{aligned}
& x+v_{x}+d x=\frac{L_{1} X+L_{2} Y+L_{3} Z+L_{4}}{L_{9} X+L_{10} Y+L_{11} Z+1} \\
& y+v_{y}+d y=\frac{L_{5} X+L_{6} Y+L_{7} Z+L_{8}}{L_{9} X+L_{10} Y+L_{11} Z+1}
\end{aligned}
$$

where:

$v_{x}, v_{y}$ - image coordinate residuals, $d x, d y$-systematic image and sensor errors,

$L_{1} \ldots L_{\mathrm{ll}}$ - coefficients of transformations

The eqn. (2) are solved for the $L$ coefficients. Each camera must be positioned, what means that both their positions and angles in space (parameters of exterior orientation) and some parameters of interior orientation (focal length, coordinates of principal point, distortions coefficients) must be calculated. One should mark at least $6 x$ control points on each image, in order to solve the eqn. (3). Finally this set of last equations may be rewritten for unknown quantities $L$ in the form:

$$
\bar{V}+\bar{b} * \bar{L}+\bar{c}=0
$$

where:

$$
\begin{aligned}
& \bar{V}=\left[\begin{array}{llll}
v_{0} & v_{1} & \ldots & v_{i-1}
\end{array}\right]^{T} \\
& v_{k}=\left[\begin{array}{ll}
v_{x k} & v_{y k}
\end{array}\right]^{T} \\
& \bar{L}=\left[\begin{array}{llll}
L_{1} & L_{2} & \ldots & L_{11}
\end{array}\right]^{T}
\end{aligned}
$$

$\bar{b} \cdot \bar{c}$ - matrixes of coefficients obtained from transformation of eqn. (2) to eqn. (3),

$k$ - ndex point's number

We have to find the $L$-vector assuring the minimum value of correction vector $V$. Best way of calculating it for any number of control points is to use the least square method as presented in eqn. (4)

$$
\frac{d\left(\bar{V}^{T} * p^{*} \bar{V}\right)}{d \bar{L}}=\bar{B}^{r} * \bar{p}^{*} \bar{B} * \bar{L}+\bar{B}^{*} \bar{p}^{*} \bar{c}=0
$$

After few (usually 5-10) iterations this equation gives solution for the $L$ coefficients. The final correction vector $V$ gives some information about the image distortions made by the camera.

The equations above are solved with the use of the least square method. The set of 3D coordinates of given point is a result. This set of equations may be written for more than two cameras, while the numerical approach remains the same.

\section{Emissivity and temperature correction}

The temperature measured by the thermal camera on some part of object's surface depends on the angles between the normal to this surface and the camera axis. The measured surfaced may be marked by 3 points, providing a surface's equation:

$$
A X+B Y+C Z+D=0
$$

An angle between camera's axis and the normal to the surface may be calculated from eqn. (6): 


$$
\cos \eta=\frac{\left[\begin{array}{lll}
A & B & C
\end{array}\right]^{-1}\left[\begin{array}{lll}
0 & 0 & 1
\end{array}\right]^{r}}{\|\left[\begin{array}{lll}
A & B & C
\end{array}\right]}
$$

The real flux emitted by a piece of surface may be finally corrected as below.

$$
\Phi=\frac{\Phi_{0}}{\cos \eta}
$$

where: $\Phi_{0}, \Phi$ denote the flux measured by the camera, and the one normal to the surface, respectively.

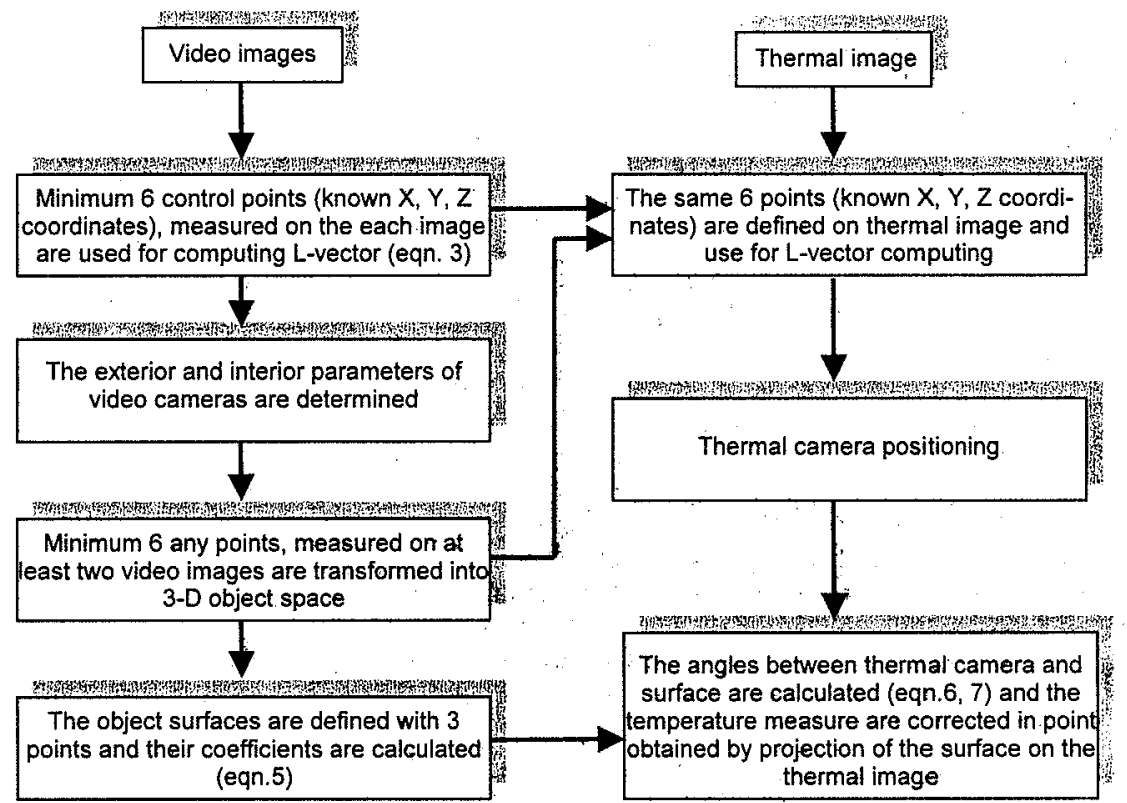

Fig.2. Flow of data in the algorithm

\section{Experimental results}

We verified the algorithm performing two examples. In the first one, a set of photogrammetric photos were taken to compare the results of the algorithm with photogrametic measurements. In the second example we corrected directional emissivity on the thermal image of a radiator for electronic device.

\section{Results of 3D points positioning}

We captured 2 video photos of close range object - computer monitor with 6 signalized control points and any 11 pass points on as shown in Fig. 3 . The distance between the object and the cameras were approximately $2 \mathrm{~m}$. The image measurement accuracy corresponds to 0.5 of the pixel spacing. The resulting average accuracy of photogrammetric solution is equivalent to a relative accuracy of $1: 4000$ of recording distance. The obtained results for the next directional emissivity correction can be accpted as very high. 
http://dx.doi.org/10.21611/qirt.1998.052

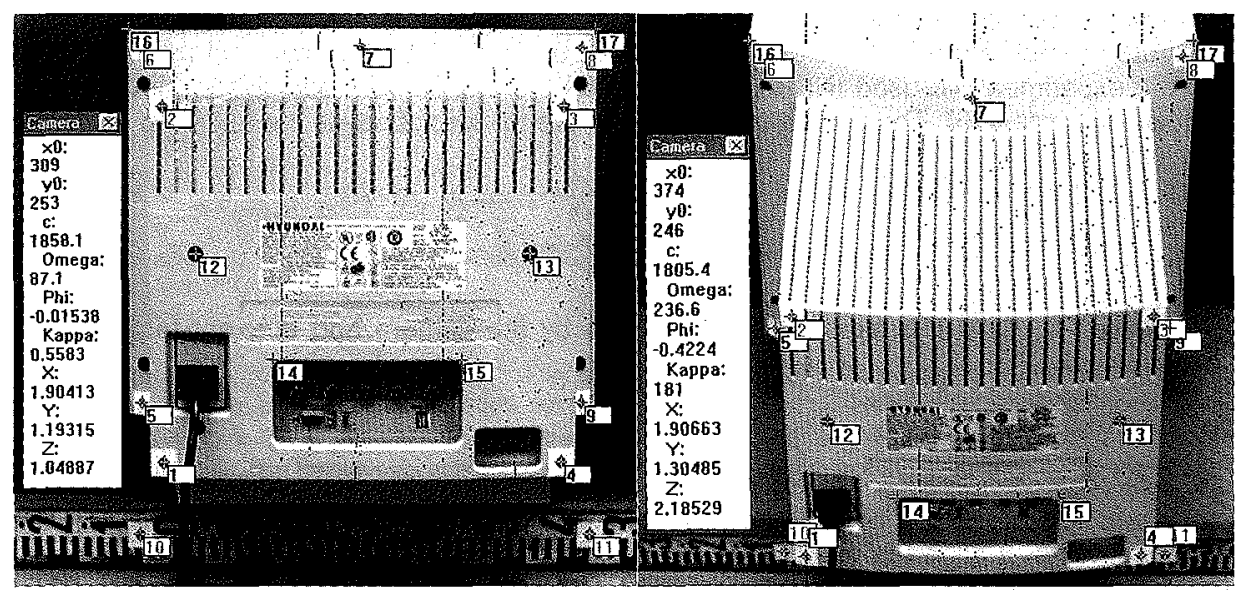

Fig. 3. Two video images of some object with 17 target points

TABLE 1. Results of 3D points positioning

\begin{tabular}{|c|c|c|c|}
\hline Point ID & $X[\mathrm{~m}]$ & $Y[\mathrm{~m}]$ & $Z[\mathrm{~m}]$ \\
\hline \multicolumn{4}{|c|}{ 3D Control Points Coordinates } \\
\hline 2 & 1.7715 & 2.8925 & 1.1060 \\
\hline 3 & 2.0655 & 2.8910 & 1.1065 \\
\hline 6 & 1.7280 & 3.2165 & 1.1670 \\
\hline 8 & 2.1120 & 3.2140 & 1.1670 \\
\hline 10 & 1.7450 & 2.9905 & 0.7845 \\
\hline 11 & 2.0955 & 2.9835 & 0.7845 \\
\hline Final Computed 3D Pass Points Coordinates \\
\hline 1 & 1.7705 & 2.9070 & 0.8485 \\
\hline 4 & 2.0655 & 2.9055 & 0.8490 \\
\hline 5 & 1.7260 & 3.2310 & 0.8620 \\
\hline 7 & 1.9185 & 3.1430 & 1.1635 \\
\hline 9 & 2.1160 & 3.2290 & 0.8625 \\
\hline 12 & 1.7955 & 2.8965 & 1.0005 \\
\hline 13 & 2.0410 & 2.8940 & 1.0010 \\
\hline 14 & 1.8520 & 2.8920 & 0.9240 \\
\hline 15 & 1.9900 & 2.8900 & 0.9245 \\
\hline 16 & 1.7180 & 3.2170 & 1.1810 \\
\hline 17 & 2.1220 & 3.2140 & 1.1820 \\
\hline
\end{tabular}

\section{Results of thermal measurements correction}

This algorithm was used for improving accuracy in temperature measurements in electronics. A hybrid resistor on the ceramic substrate was a heat source taken into account. The temperature was corrected according to angle of viewing as in Fig. 4.
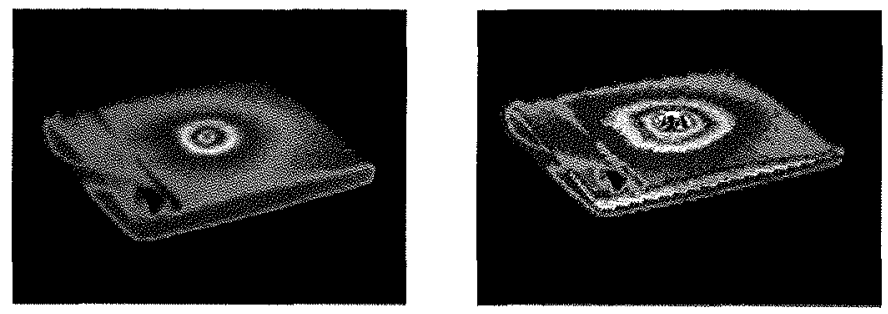

Fig 4. Corrected temperature on the hybrid circuit 
Typical thermal camera produces a data corresponding to the radiation, not the computed temperature. Temperature is obtained using a calibration curve in the computer. For the experiment we used INFRAMETRICS 760 thermal camera equipped with the digital acquisition system [6]. The correction of temperature for black body is the following (after the eqn. (7)):

$$
T=\sqrt[4]{\frac{T_{0}^{4}-T_{a}^{4}}{\cos \varphi}+T_{a}^{4}}
$$

where: $T_{0}, T_{a}$ is the temperature measured by the camera and ambient temperature, respectively.

\section{Conclusions}

The method presented in this paper gives the ability to reconstruct $3 \mathrm{D}$ coordinates of measured close range object, including the positions of all cameras used for the measurements. It allows computing both the angles between camera's axes and normal to the measured surface and the distance between the camera and the object. This is necessary for the correction of emissivity and in consequence the temperature measured by camera. Although the method presented in this paper is based on well-known algorithms, a few new improvements were added to include thermal system for photogrammetric application. We do not need to declare the real coordinates of reference points on thermal image what it is a major modification and simplification of existing methods. The algorithm presented is useful in various applications, e.g.: in photogrammetric engineering measurements, industrial metrology, robotics, etc.

\section{REFERENCES}

[1] ABDEL-AZIZ (Y.I.), KARARA (H. M.) Direct Linear Transformation from Comparator Coordinates into Object-space Coordinates in Close Range Photogrametry. Proceedings of ASP Symposium on Close Range Photo-grammetry, Urbana, Illinios, January 1971.

[2] HOBROUGHT (T.B.) - A Future for Realtime Photogrammetry. Memsuration, Photogrametrie, Genie Rural \$/85 p.312-315.

[3] SAWICKI (P.), WIĘCEK (B.) - CCD Multicameras interactive digital system for close range photogrammetric applications. International Archives of Photogrammetry and Remote Sensing, 1996 Vol. XXXI, Part B5, pp. 512-517.

[4] SAWICKI (P.), WIECCEK (B.) - Cyfrowy system video termalny dla aplikacji w bliskim zasiegu. Sympozjum PTFiT. Obrazy cyfrowe $w$ fotogrametrii, teledetekcji oraz GIS. Kraków 17.06.1998, Archiwum Fotogrametrii, Kartografii i Teledetekcji Vol. 8, s.13/1-13/8 (in Polish)

[5] SITEK (Z.) - Fotogrametria ogólna i inżynieryjna, PPWK 1991 (in Polish)

[6] WIĘCEK (B.); SAWICKI (P.), STEIN (R.) - Directional emissivity correction, III Workshop on "Advances in Signal Processing for Non Destructive Testing", August 5-8, 1997, Quebec, Canada. 